\title{
On the Performance of Linear Least-Squares Estimation in Wireless Positioning Systems
}

\author{
Sinan Gezici* ${ }^{*}$ Ismail Guvenc ${ }^{\sharp}$, and Zafer Sahinoglu ${ }^{\S}$ \\ * Department of Electrical and Electronics Engineering, Bilkent University, Bilkent, Ankara 06800, Turkey \\ $\sharp$ DoCoMo Communications Laboratories USA, Inc., 3240 Hillview Avenue, Palo Alto, CA 94304, USA \\ $\S$ Mitsubishi Electric Research Laboratories, 201 Broadway Avenue, Cambridge, MA 02139, USA \\ E-mails: gezici@ieee.org,iguvenc@docomolabs-usa.com,zafer@merl.com
}

\begin{abstract}
A common technique for wireless positioning is to estimate time-of-arrivals (TOAs) of signals traveling between a target node and a number of reference nodes, and then to determine the position of the target node based on those TOA parameters. In determining the position of the target node from TOA parameters, linear or nonlinear least-squares (LS) estimation techniques can be employed. Although the linear LS techniques are suboptimal in general, they facilitate lowcomplexity position estimation. In this paper, performance of various linear LS techniques are compared, and suboptimality of the linear approach is quantified in terms of the Cramer-Rao lower bound (CRLB). Simulations are performed to compare the performance of the linear LS approaches versus the CRLBs for linear and nonlinear techniques.
\end{abstract}

Index Terms - Wireless positioning, time-of-arrival (TOA), least-squares (LS) estimation, Cramer-Rao lower bound (CRLB).

\section{INTRODUCTION}

The subject of positioning in wireless systems has been drawing considerable attention due to its potential applications and services for both cellular and short-range systems [1], [2]. For cellular systems, enhanced-911, improved fraud detection, location sensitive billing, intelligent transport systems and improved traffic management can become feasible with accurate positioning [1], [3]. On the other hand, for short-range networks, position estimation facilitates applications such as inventory tracking, intruder detection, tracking of fire-fighters and miners, home automation and patient monitoring [4], [5]. These potential applications of wireless positioning were also recognized by the IEEE, which approved a new amendment, IEEE 802.15.4a, that provides a new physical layer for low data rate communications combined with positioning capabilities [6]-[8]. Also, the Federal Communications Commission (FCC) in the U.S. required wireless providers to estimate the position of mobile users within tens of meters for emergency 911 calls [9].

A common approach in wireless positioning systems involves estimation of position in two steps. In the first step, position related parameters, such as time-of-arrivals (TOAs) of signals traveling between the target node, i.e. the node to be located, and a number of reference nodes are estimated. Then, in the second step, the position is estimated based on the signal

\footnotetext{
${ }^{0}$ This research was supported in part by the EU FP7 Project NEWCOM++ under grant no. 216715.
}

parameters obtained in the first step [3]. The position related parameters estimated in the first step are commonly TOA or received signal strength (RSS) parameters, which provide an estimate for the distance between two nodes, or angle-ofarrival (AOA) parameters, which estimate the angles between the nodes [1]. For distance based positioning algorithms, such as TOA or RSS based schemes, the maximum likelihood (ML) solution can be obtained by a nonlinear least-squares (N-LS) approach, under certain conditions [10]. The N-LS approach requires the minimization of a cost function that requires numerical search methods such as the steepest descent or the Gauss-Newton techniques. Such techniques can have high computational complexity and they typically require good initialization in order to avoid converging to the local minima of the cost function [11].

In order to obtain a closed-form solution and avoid computational complexity of the N-LS approach, the set of expressions corresponding to the position related parameter estimates can be linearized using the Taylor series expansion [12]. However, such an approach still requires an intermediate position estimate to obtain the Jacobian matrix, which should be sufficiently close to the true position of the target node for the linearity assumption to hold. Alternatively, a linear leastsquares (L-LS) approach based on the measured distances was initially proposed in [13]. In the L-LS approach, the expression corresponding to one of the reference nodes is subtracted from all the other expressions in order to obtain linear relations in terms of the target node position. Various versions of the L-LS approach are studied in [14] and [15], which subtract different expressions or average of them from the remaining expressions.

The L-LS approach is a suboptimal positioning technique, which provides a solution with low computational complexity. Therefore, it can be employed for applications that require low cost/complexity implementation with reasonable positioning accuracy. In addition, for applications that require precise position estimation, L-LS approaches can be used to obtain an initial position estimate for initializing high-accuracy positioning algorithms, such as the N-LS approach and linearization based on the Taylor series [16]. A good initialization can significantly decrease the computational complexity and the final localization error of a high-accuracy technique. Therefore, performance analysis of the L-LS approaches is important from multiple perspectives. 
Although theoretical mean square errors (MSEs) of the LLS approach are derived for various scenarios in [17], no studies have considered the theoretical lower bounds that can be achieved when using linearized measurements. In this paper, performance of L-LS techniques for position estimation is investigated. Specifically, a closed-form approximate expression for the Cramer-Rao lower bound (CRLB) is obtained for position estimation based on linearized measurements. Then, it is shown that the bound applies to various L-LS approaches proposed in the literature. In addition, the performance of the L-LS algorithms is compared against each other and the CRLBs for the linear and nonlinear models. Practical implications of the performance analysis are investigated.

The remainder of the paper is organized as follows. In Section II, the system model is defined, and measurement models are introduced. Then, the N-LS estimation and its CRLB are briefly reviewed in Section III. In Section IV, the L-LS approaches are studied and their CRLB is derived. Finally, the simulation results are presented in Section V, and concluding remarks are made in Section VI.

\section{SYSTEM MOdEL}

Consider a wireless network with $N$ reference nodes, with the $i$ th node being located at $\boldsymbol{l}_{i}=\left[\begin{array}{ll}x_{i} & y_{i}\end{array}\right]^{T}$ for $i=1, \ldots, N$. The aim is to estimate the position of the target node, denoted by $\boldsymbol{l}=\left[\begin{array}{ll}x & y\end{array}\right]^{T}$, based on $N$ TOA measurements between the target node and the reference nodes ${ }^{1}$.

Let $z_{i}$ represent the distance estimate obtained from the $i$ th TOA measurement:

$$
z_{i}=c \tau_{i}=f_{i}(x, y)+n_{i}, \quad i=1, \ldots, N
$$

where $\tau_{i}$ represents the TOA estimate for the $i$ th signal, $c$ is the speed of light, $n_{i}$ is the noise in the $i$ th measurement, and $f_{i}(x, y)$ is the true distance between the target node and the $i$ th reference node, given by

$$
f_{i}(x, y)=\sqrt{\left(x-x_{i}\right)^{2}+\left(y-y_{i}\right)^{2}} .
$$

Commonly, the noise is modeled by a zero-mean Gaussian random variable, when the reference node and the target node has direct line-of-sight (LOS). However, in non-line-ofsight (NLOS) conditions, the noise distribution can be quite different from a Gaussian distribution [18], [19].

In the absence of noise in the system, each TOA measurement specifies a circle for the possible positions of the target node, and the intersection of those circles determines the target position [1]. This geometric technique called, trilateration, yields ambiguous solutions in the presence of noise in the system, since the circles defined by the TOA measurements may intersect at multiple points due erroneous TOA estimation, as shown in Fig. 1.

Due to the limitations of the geometric technique, statistical approaches are commonly employed in wireless positioning systems [3]. A popular statistical positioning algorithm is the N-LS technique studied below.

\footnotetext{
${ }^{1}$ In fact, the results can be applied to any systems that estimate distances between nodes, such as a received signal strength (RSS) based positioning system, which performs distance estimation based on received signal energy [1].
}

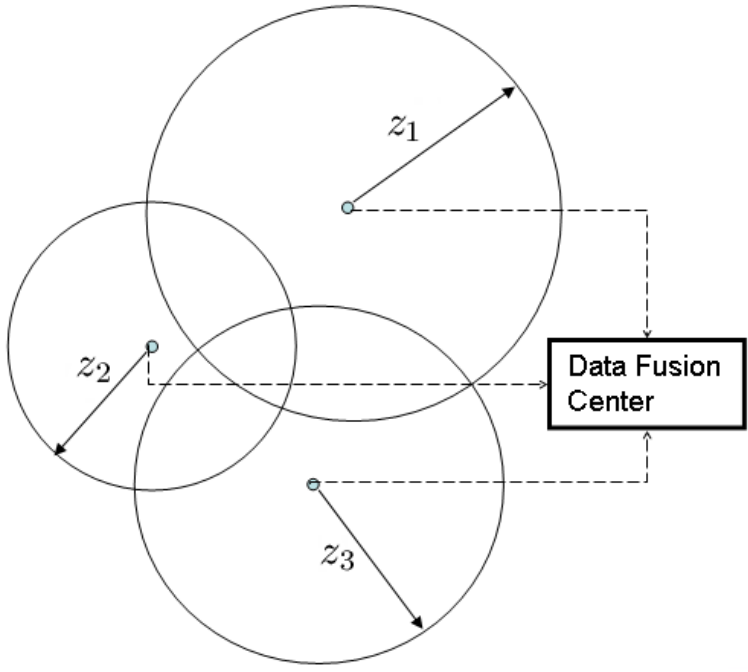

Fig. 1. Trilateration yields multiple intersection of circles defined by TOA measurements in the presence of noise.

\section{NONLINEAR LS ESTIMATION}

Given a set of distance, or commonly called "range", estimates as given by (1), an N-LS estimator calculates the position of the target node as follows:

$$
\hat{\boldsymbol{l}}=\left[\begin{array}{ll}
\hat{x} & \hat{y}
\end{array}\right]=\arg \min _{(x, y)} \sum_{i=1}^{N} \beta_{i}\left(z_{i}-f_{i}(x, y)\right)^{2}
$$

where $\beta_{i}$ represents a weighting coefficient for the $i$ measurement, which commonly reflects the reliability of the measurement (estimation).

It is well-known that the N-LS solution in (3) corresponds to the ML estimator of the target position for independent noise components, each distributed as $^{2} n_{i} \sim \mathcal{N}\left(0, \sigma_{i}^{2}\right)$, and for the weighting coefficient $\beta_{i}$ being given by $1 / \sigma_{i}^{2}$ for $i=$ $1, \ldots, N$ [3]. Since an ML estimator asymptotically achieves the Cramer-Rao lower bound (CRLB), the N-LS estimator provides an asymptotically optimal estimator under the stated conditions.

For the model in (1) with independent zero-mean Gaussian noise components, the CRLB for an unbiased N-LS estimator $\hat{l}$ can be expressed as [20], [21]

$$
\operatorname{Cov}\{\hat{\boldsymbol{l}}\} \geq \mathbf{I}^{-1}
$$

with the Fisher information matrix (FIM) being given by

$$
\mathbf{I}=\left[\begin{array}{cc}
\sum_{i=1}^{N} \frac{\left(x-x_{i}\right)^{2}}{\sigma_{i}^{2} f_{i}^{2}(x, y)} & \sum_{i=1}^{N} \frac{\left(x-x_{i}\right)\left(y-y_{i}\right)}{\sigma_{i}^{2} f_{i}^{2}(x, y)} \\
\sum_{i=1}^{N} \frac{\left(x-x_{i}\right)\left(y-y_{i}\right)}{\sigma_{i}^{2} f_{i}^{2}(x, y)} & \sum_{i=1}^{N} \frac{\left(y-y_{i}\right)^{2}}{\sigma_{i}^{2} f_{i}^{2}(x, y)}
\end{array}\right]
$$

where $\sigma_{i}^{2}$ denotes the variance of the noise in the $i$ th measurement. Then, the lower bound on the mean-square error (MSE) can be obtained as

$$
\operatorname{MSE}=\mathrm{E}\left\{\|\hat{\boldsymbol{l}}-\boldsymbol{l}\|^{2}\right\} \geq \operatorname{trace}\left\{\mathbf{I}^{-1}\right\}=\frac{\mathrm{I}_{11}+\mathrm{I}_{22}}{\mathrm{I}_{11} \mathrm{I}_{22}-\mathrm{I}_{12}^{2}}
$$

${ }^{2} \mathcal{N}\left(\mu, \sigma^{2}\right)$ represents a Gaussian random variable with mean $\mu$ and variance $\sigma^{2}$. 
where $\mathrm{I}_{i j}$ represents the element of $\mathbf{I}$ in the $i$ th row and $j$ th column. From (5), $\mathrm{I}_{11}+\mathrm{I}_{22}$ can be shown to be equal to $\sum_{i=1}^{N} \sigma_{i}^{-2}$.

Since the N-LS estimator in (3) can asymptotically achieve the minimum MSE (MMSE) in (6) under certain conditions, it provides a benchmark for the performance of other estimators. The main disadvantage of the N-LS estimator is related to the nonlinear nature of the optimization problem, which increases its computational complexity. The common techniques for obtaining the N-LS estimator in (3) include gradient descent algorithms and linearization techniques via the Taylor series expansion [1], [12].

\section{LINEAR LS APPROACH AND CRLB ANALYSIS}

\section{A. Linear LS Estimation}

An alternative approach to the N-LS estimation is the LLS approach [13]-[15], [22]. In an L-LS technique, a new measurement set is obtained from the measurements in (1) by certain operations that result in linear relations.

The L-LS approach starts with the following set of equations

$$
z_{i}^{2}=\left(x-x_{i}\right)^{2}+\left(y-y_{i}\right)^{2}, \quad \text { for } i=1, \ldots, N,
$$

where each distance measurement is assumed to define a circle of uncertain region [22]. Then, one of the equations in (7), say the $r$ th one, is fixed and subtracted from all of the other equations. After some manipulation, the following linear relation can be obtained [22]:

$$
\mathbf{A} l=\mathbf{p},
$$

where $\boldsymbol{l}=\left[\begin{array}{ll}x & y\end{array}\right]^{T}$,

$$
\mathbf{A}=2\left[\begin{array}{cc}
x_{1}-x_{r} & y_{1}-y_{r} \\
\vdots & \vdots \\
x_{r-1}-x_{r} & y_{r-1}-y_{r} \\
x_{r+1}-x_{r} & y_{r+1}-y_{r} \\
\vdots & \vdots \\
x_{N}-x_{r} & y_{N}-y_{r}
\end{array}\right],
$$

and

$$
\mathbf{p}=\left[\begin{array}{c}
z_{r}^{2}-z_{1}^{2}-k_{r}+k_{1} \\
\vdots \\
z_{r}^{2}-z_{r-1}^{2}-k_{r}+k_{r-1} \\
z_{r}^{2}-z_{r+1}^{2}-k_{r}+k_{r+1} \\
\vdots \\
z_{r}^{2}-z_{N}^{2}-k_{r}+k_{N}
\end{array}\right]
$$

with

$$
k_{i}=x_{i}^{2}+y_{i}^{2},
$$

for $i=1,2, \ldots, N$, and $r$ being the selected reference node index that is used to obtain linear relations. Note that $\mathbf{A}$ is an $(N-1) \times 2$ matrix, and $\mathbf{p}$ is a vector of size $(N-1)$, since the $r$ th measurement is used as a reference for the other measurements.

From (8), the LS solution can be obtained as

$$
\hat{\boldsymbol{l}}=\left(\mathbf{A}^{T} \mathbf{A}\right)^{-1} \mathbf{A}^{T} \mathbf{p} .
$$

This estimator is called the linear LS (L-LS) estimator. Compared to the N-LS estimator in (3), it has low computational complexity. However, it is suboptimal in general, and the amount of its suboptimality can be quantified in terms the CRLB.

\section{B. CRLB Analysis}

In order to derive the CRLB for the L-LS approach, we first make the observation from (8)-(12) that the L-LS algorithm utilizes the measurements $z_{i}, i=1, \ldots, N$, only through the terms $z_{r}^{2}-z_{i}^{2}$, for $i=1, \ldots, N$ and $i \neq r$. Therefore, the measurement set for the L-LS algorithm effectively becomes

$$
\tilde{z}_{i}=z_{r}^{2}-z_{\tilde{i}}^{2}
$$

for $i=1, \ldots, N-1$, where

$$
\tilde{i}=\left\{\begin{array}{ll}
i, & i<r \\
i+1, & i \geq r
\end{array} .\right.
$$

Let $r=N$ without loss of generality and $\tilde{\mathbf{z}}$ represent the vector consisting of $\tilde{z}_{i}$ 's in (13); i.e.,

$$
\tilde{\mathbf{z}}=\left[\begin{array}{lll}
z_{N}^{2}-z_{1}^{2} & z_{N}^{2}-z_{2}^{2} \cdots z_{N}^{2}-z_{N-1}^{2}
\end{array}\right] .
$$

In order to calculate the CRLB for any unbiased estimator that employs the observation (measurement set) $\tilde{\mathbf{z}}$, we first need to obtain the conditional probability density function (PDF) of $\tilde{\mathbf{z}}$ given the location of the target node $\boldsymbol{l}$.

From (1), (2) and (13), $\tilde{z}_{i}$ can be expressed as

$$
\begin{aligned}
\tilde{z}_{i} & =k_{N}-k_{i}+2\left(x_{i}-x_{N}\right) x+2\left(y_{i}-y_{N}\right) y \\
& +2 n_{N} f_{N}(x, y)-2 n_{i} f_{i}(x, y)+\left(n_{N}^{2}-n_{i}^{2}\right),
\end{aligned}
$$

for $i=1, \ldots, N-1$, where $k_{i}$ and $f_{i}(x, y)$ are as in (11) and (2), respectively. In order to obtain a closed-form CRLB expression, the last term in (16), namely $n_{N}^{2}-n_{i}^{2}$, is modeled as a Gaussian random variable. In other words, conditioned on $\boldsymbol{l}=\left[\begin{array}{ll}x & y\end{array}\right]^{T}, \tilde{z}_{i}$ in (16) is approximated by a Gaussian distribution. This approximation gets quite accurate when the noise variance is considerably smaller than the distances between the nodes. In general, it is expected that the CRLB to be obtained under this approximate model provides a smaller bound than the exact model. In other words, a smaller CRLB is obtained by the Gaussian approximation, but the difference between the approximate and the exact models diminishes for small noise terms.

According to the model in (16), the conditional PDF of $\tilde{z}_{i}$ given $\boldsymbol{l}=\left[\begin{array}{ll}x & y\end{array}\right]^{T}$ can be obtained, after some manipulation, as

$$
\tilde{z}_{i} \mid \boldsymbol{l} \sim \mathcal{N}\left(\mu_{i}(x, y), \tilde{\sigma}_{i}(x, y)\right)
$$

where

$$
\begin{aligned}
& \mu_{i}(x, y)=f_{N}^{2}(x, y)-f_{i}^{2}(x, y)+\sigma_{N}^{2}-\sigma_{i}^{2} \\
& \tilde{\sigma}_{i}(x, y)=4\left[\sigma_{N}^{2} f_{N}^{2}(x, y)+\sigma_{i}^{2} f_{i}^{2}(x, y)\right]+2\left(\sigma_{N}^{4}+\sigma_{i}^{4}\right) .
\end{aligned}
$$


In addition, the covariance terms can be calculated as ${ }^{3}$

$$
\mathrm{E}\left\{\left(\tilde{z}_{i}-\mu_{i}\right)\left(\tilde{z}_{j}-\mu_{j}\right)\right\}=4 \sigma_{N}^{2} f_{N}^{2}(x, y)+2 \sigma_{N}^{4}
$$

for $i \neq j$. Then, the conditional distribution of $\tilde{\mathbf{z}}$ given $l$ can be expressed as

$$
\tilde{\mathbf{z}} \mid \boldsymbol{l} \sim \mathcal{N}(\boldsymbol{\mu}(x, y), \boldsymbol{\Sigma}(x, y)),
$$

where

$$
\boldsymbol{\mu}(x, y)=\left[\begin{array}{c}
\mu_{1}(x, y) \\
\mu_{2}(x, y) \\
\vdots \\
\mu_{N-1}(x, y)
\end{array}\right],
$$

with $\mu_{i}(x, y)$ being given by (18) for $i=1, \ldots, N-1$, and

$$
\begin{aligned}
& \boldsymbol{\Sigma}(x, y)=\left(4 \sigma_{N}^{2} f_{N}^{2}(x, y)+2 \sigma_{N}^{4}\right) \mathbf{1}_{N-1} \\
& +2 \operatorname{diag}\left\{2 \sigma_{1}^{2} f_{1}^{2}(x, y)+\sigma_{1}^{4}, \ldots, 2 \sigma_{N-1}^{2} f_{N-1}^{2}(x, y)+\sigma_{N-1}^{4}\right\}
\end{aligned}
$$

with $\mathbf{1}_{N-1}$ representing an $(N-1) \times(N-1)$ matrix of ones, and $\operatorname{diag}\left\{a_{1}, \ldots, a_{M}\right\}$ denoting an $M \times M$ diagonal matrix with $a_{i}$ being the $i$ th diagonal.

From the signal model given by (21)-(23), the CRLB can be obtained as stated in the following proposition.

Proposition 1: The CRLB on the MSE of an unbiased position estimator $\hat{l}$ based on the measurements in (21) is given by

$$
\mathrm{E}\left\{\|\hat{\boldsymbol{l}}-\boldsymbol{l}\|^{2}\right\} \geq \frac{\tilde{\mathrm{I}}_{11}+\tilde{\mathrm{I}}_{22}}{\tilde{\mathrm{I}}_{11} \tilde{\mathrm{I}}_{22}-\tilde{\mathrm{I}}_{12}^{2}},
$$

where $^{4}$

$$
\begin{aligned}
\tilde{\mathrm{I}}_{11} & =\frac{(N-1)}{2 g^{2}}\left[g \frac{\partial^{2} g}{\partial x^{2}}-\left(\frac{\partial g}{\partial x}\right)^{2}\right]+4 \mathbf{b}_{x}^{T} \boldsymbol{\Sigma}^{-1} \mathbf{b}_{x} \\
& +2 \sigma_{N}^{2} f_{N}^{2} \sum_{i, j=1}^{N-1} \frac{\partial^{2} h_{i j}}{\partial x^{2}}+2 \sum_{i=1}^{N-1} \sigma_{i}^{2} f_{i}^{2} \frac{\partial^{2} h_{i i}}{\partial x^{2}} \\
\tilde{\mathrm{I}}_{22} & =\frac{(N-1)}{2 g^{2}}\left[g \frac{\partial^{2} g}{\partial y^{2}}-\left(\frac{\partial g}{\partial y}\right)^{2}\right]+4 \mathbf{b}_{y}^{T} \boldsymbol{\Sigma}^{-1} \mathbf{b}_{y} \\
& +2 \sigma_{N}^{2} f_{N}^{2} \sum_{i, j=1}^{N-1} \frac{\partial^{2} h_{i j}}{\partial y^{2}}+2 \sum_{i=1}^{N-1} \sigma_{i}^{2} f_{i}^{2} \frac{\partial^{2} h_{i i}}{\partial y^{2}} \\
\tilde{\mathrm{I}}_{12} & =\frac{(N-1)}{2 g^{2}}\left[g \frac{\partial^{2} g}{\partial x \partial y}-\frac{\partial g}{\partial x} \frac{\partial g}{\partial y}\right]+4 \mathbf{b}_{x}^{T} \boldsymbol{\Sigma}^{-1} \mathbf{b}_{y} \\
& +2 \sigma_{N}^{2} f_{N}^{2} \sum_{i, j=1}^{N-1} \frac{\partial^{2} h_{i j}}{\partial x \partial y}+2 \sum_{i=1}^{N-1} \sigma_{i}^{2} f_{i}^{2} \frac{\partial^{2} h_{i i}}{\partial x \partial y}
\end{aligned}
$$

with $\boldsymbol{\Sigma}(x, y)$ being given by (23), $g(x, y) \doteq|\boldsymbol{\Sigma}(x, y)|$, $h_{i j}(x, y) \doteq\left[\boldsymbol{\Sigma}^{-1}(x, y)\right]_{i j}, \mathbf{b}_{x} \doteq\left[x_{1}-x_{N} \cdots x_{N-1}-x_{N}\right]^{T}$ and $\mathbf{b}_{y} \doteq\left[y_{1}-y_{N} \cdots y_{N-1}-y_{N}\right]^{T}$.

Proof: Please see Appendix A.

Proposition 1 provides generic expressions to evaluate the CRLB for any positioning system configuration. In Section V,

\footnotetext{
${ }^{3}(x, y)$ is dropped from $\mu_{i}(x, y)$ for convenience.

${ }^{4}(x, y)$ 's are omitted in order to have simpler expressions.
}

the expressions in Proposition 1 will be employed to obtain CRLBs for various scenarios. Although the expressions in (25)-(27) seem complicated, they provide a closed-form CRLB expression that can be easily evaluated by using computer programs, such as Matlab ${ }^{5}$.

\section{Other Linear LS Techniques}

The L-LS technique studied in Section IV-A, call it $L-L S$ 1 , selects one of the equations related to one of the reference nodes, and subtracts it from all the other equations to obtain $N-1$ linear relations, where $N$ is the number of reference nodes. Another L-LS approach (call it $L-L S$-2) obtains $\left(\begin{array}{c}N \\ 2\end{array}\right)$ linear equations by subtracting each equation from all of the other equations [14], [13]. Similar to $L-L S-1$, the linear LS solution is obtained for the position of the target node in the $L-L S-2$ technique.

In the $L-L S-2$ technique, the following observations are employed for position estimation:

$$
\check{z}_{i j}=z_{i}^{2}-z_{j}^{2}, \quad i, j=1,2, \ldots, N, i<j .
$$

Comparison of the measurements in (28) with those in (13) reveals that all the additional measurements in (28) can in fact be obtained from the ones in (13) by simple subtraction operations. In other words, there is no independent observation in the measurement set for the $L-L S-2$ technique compared to that for the $L-L S-1$ technique. Therefore, the CRLB for the $L-L S-1$ technique is also valid for the the $L-L S-2$.

In another L-LS technique (call it $L-L S-3$ ), instead of obtaining the difference of the equations directly as in the $L-L S$ 1 and $L-L S-2$ approaches, the average of the measurements is obtained first, and this average is subtracted from all the equations by resulting in $N$ linear relations [15]. Then, the linear LS solution is obtained for the position of the target node.

The observation set employed in the $L-L S-3$ technique can be expressed as

$$
\bar{z}_{i}=z_{i}^{2}-\frac{1}{N} \sum_{j=1}^{N} z_{j}^{2}, \quad i=1,2, \ldots, N .
$$

Although this observation set seems quite different from the one in (13), it can be shown that each measurement in one set is dependent on a number of measurements in the other set.

Proposition 2: The CRLB for estimating the position of a target node based on measurements in (29) is the same as the CRLB based on measurements in (13).

Proof: First, it can observed that each measurement in (13) is simply equal to the difference of two measurements in (29). In other words, $\tilde{z}_{i}=\bar{z}_{r}-\bar{z}_{\tilde{i}}$ for $i=1, \ldots, N-1$, where $\tilde{i}$ is as in (14).

On the other hand, if the average of all the measurements in (13) is taken, the $r$ th measurement in (29) is obtained; i.e.,

$$
\frac{1}{N} \sum_{i=1}^{N-1} \tilde{z}_{i}=\frac{1}{N} \sum_{\substack{i=1 \\ i \neq r}}^{N}\left(z_{r}^{2}-z_{i}^{2}\right)=z_{r}^{2}-\frac{1}{N} \sum_{i=1}^{N} z_{i}^{2}=\bar{z}_{r} .
$$

\footnotetext{
${ }^{5}$ Especially, the symbolic toolbox facilitates easy evaluation of the CRLB
} 


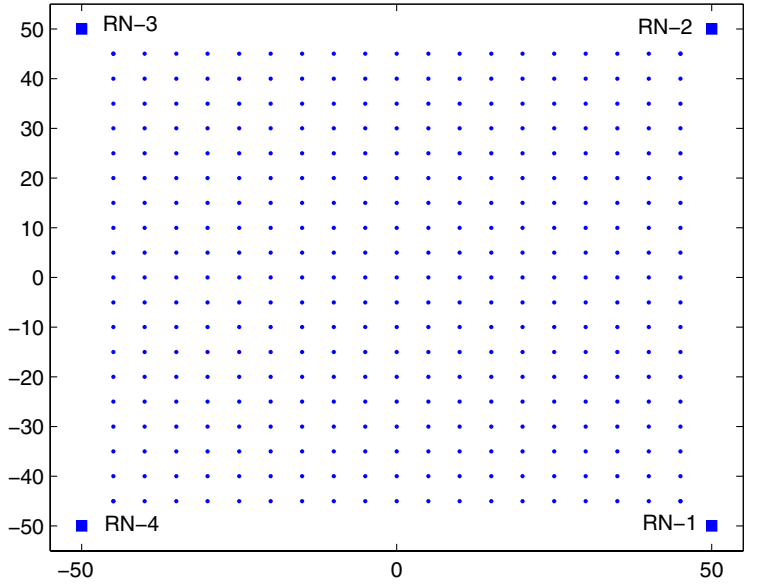

Fig. 2. Simulation environment with 4 reference nodes (RNs), where the coordinates are in the unit of meters.

Then, the subtraction of each $\tilde{z}_{i}$ from (30) yields the remaining measurements in (29), since $\bar{z}_{i}=\bar{z}_{r}-\left(z_{r}^{2}-z_{i}^{2}\right)$ for $i=$ $1, \ldots, N, i \neq r$.

Since the measurements in the sets (13) and (29) are dependent on each other, they carry the same amount of information, hence result in the same CRLB.

\section{Simulation Results}

In this section, simulations are performed in order to evaluate the CRLBs and compare the performance of the three L-LS algorithms studied in the previous section. In the simulation environment, there are 4 reference nodes involved in position estimation, which are located on the corners of a square, as illustrated in Fig. 2. In order to calculate the performance of the L-LS algorithms, the target node is inserted at various points in the environment, marked by small dots, and the average MSE value is calculated. In addition, for each target position, 1000 estimations are performed in order to average out the effects of noise. Similarly, average CRLBs are obtained by averaging the CRLBs at various target positions. It is assumed that the noise variances are the same at all target positions and for all reference nodes.

In Fig. 3, the square roots of the MSEs (RMSEs) are plotted for various noise levels. It is observed that there is a linear relation between the standard deviation of the noise and the RMSE, which can also be observed from (5) and (6) for the CRLB in the nonlinear case. Comparison of the three L-LS algorithms reveals that $L-L S-2$ and $L-L S-3$ have the same performance, which is better than that of $L$ $L S$-1. In other words, $L-L S-1$ has the highest RMSEs. The worst performance of $L-L S-1$ is mainly due to its estimation technique that uses one of the reference nodes as the reference for other measurements (c.f. eqn. (13)). In the presence of large noise in the reference, the estimate can have significant errors. However, $L-L S-2$ and $L-L S-3$ have an averaging effect in selecting the reference, since not only a single measurement is used as the reference (c.f. (28) and (29)).

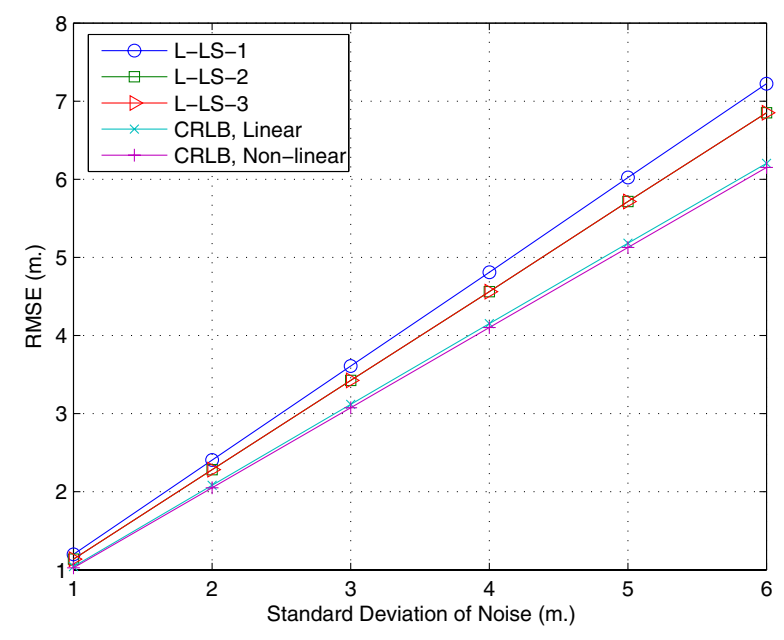

Fig. 3. RMSE versus the noise variance (equal noise variances are assumed for all nodes) for the linear LS algorithms, and the CRLBs.

In addition, there is considerable difference between the theoretical limits and the L-LS algorithms. For example, for a noise standard deviation of 6 meters, the performance difference between $L-L S-1$ and the CRLB is about 1 meter. Finally, the CRLBs for the linear and nonlinear cases in Section IV and Section III, respectively, seem to have close values, but the CRLB for the nonlinear case is lower than that for the linear case, as expected. The results seem to indicate that the linear techniques can have similar performance to nonlinear ones. However, it should be noted that the CRLB in Fig. 3 for linear techniques is approximate due to the Gaussian approximation, and its exact value can be higher, especially for large noise variances. For small noise variances, it can be concluded that it is possible to design linear techniques that have similar performance to nonlinear ones.

\section{CONCLUding REMARKS}

Linear LS estimation has been studied for TOA based positioning in wireless systems. After a brief review of the N-LS approach, the L-LS technique has been investigated. A generic CRLB expression has been obtained for positioning systems that employ linearized relations. It has been shown that the CRLB provides a lower bound for the three LLS algorithms proposed in the literature. Then, simulation results have been provided for comparison of various L-LS approaches and the CRLBs.

\section{APPENDIX}

\section{A. Proof of Proposition 1}

From (21)-(23), the log-likelihood function of $\tilde{\mathbf{z}}$ given $\boldsymbol{l}=$ $\left[\begin{array}{ll}x & y\end{array}\right]^{T}$ can be obtained as

$$
\begin{aligned}
\ln p(\tilde{\mathbf{z}} \mid \boldsymbol{l}) & \propto-\frac{(N-1)}{2} \ln |\boldsymbol{\Sigma}(x, y)| \\
& -\frac{1}{2}(\tilde{\mathbf{z}}-\boldsymbol{\mu}(x, y))^{T} \boldsymbol{\Sigma}^{-1}(x, y)(\tilde{\mathbf{z}}-\boldsymbol{\mu}(x, y)) .
\end{aligned}
$$


In order to obtain the FIM, given by

$$
\tilde{\mathbf{I}}=\left[\begin{array}{cc}
-\mathrm{E}\left\{\frac{\partial^{2}}{\partial x^{2}} \ln p(\tilde{\mathbf{z}} \mid \boldsymbol{l})\right\} & -\mathrm{E}\left\{\frac{\partial^{2}}{\partial x \partial y} \ln p(\tilde{\mathbf{z}} \mid \boldsymbol{l})\right\} \\
-\mathrm{E}\left\{\frac{\partial^{2}}{\partial x \partial y} \ln p(\tilde{\mathbf{z}} \mid \boldsymbol{l})\right\} & -\mathrm{E}\left\{\frac{\partial^{2}}{\partial y^{2}} \ln p(\tilde{\mathbf{z}} \mid \boldsymbol{l})\right\}
\end{array}\right],
$$

we first need to obtain the partial derivatives of the loglikelihood function in (31).

For simplicity of the expressions, drop the $(x, y)$ 's in (31) and let $g=|\boldsymbol{\Sigma}|$ and $h_{i j}=\left[\boldsymbol{\Sigma}^{-1}\right]_{i j}$, where $|\boldsymbol{\Sigma}|$ represent the determinant of $\boldsymbol{\Sigma}$ and $\left[\boldsymbol{\Sigma}^{-1}\right]_{i j}$ represent the element of $\boldsymbol{\Sigma}^{-1}$ in the $i$ th row and $j$ th column. Then, the first derivative of (31) with respect to $x$ can be calculated as

$$
\begin{gathered}
\frac{\partial}{\partial x} \ln p(\tilde{\mathbf{z}} \mid \boldsymbol{l})=-\frac{(N-1)}{2 g} \frac{\partial g}{\partial x}-\frac{1}{2} \sum_{i, j=1}^{N-1}\left\{-\frac{\partial \mu_{i}}{\partial x} h_{i j}\left(\tilde{z}_{j}-\mu_{j}\right)\right. \\
\left.+\left(\tilde{z}_{i}-\mu_{i}\right) \frac{\partial h_{i j}}{\partial x}\left(\tilde{z}_{j}-\mu_{j}\right)-\left(\tilde{z}_{i}-\mu_{i}\right) h_{i, j} \frac{\partial \mu_{j}}{\partial x}\right\} .
\end{gathered}
$$

For $\partial \ln p(\tilde{\mathbf{z}} \mid \boldsymbol{l}) / \partial y$, the same expression as in (33) is obtained, with only difference being that the derivatives are with respect to $y$ in that case.

After obtaining the second derivative and taking the expectation, we get

$$
\begin{aligned}
\mathrm{E}\left\{\frac{\partial^{2}}{\partial x^{2}} \ln p(\tilde{\mathbf{z}} \mid \boldsymbol{l})\right\}= & -\frac{(N-1)}{2 g^{2}}\left[g \frac{\partial^{2} g}{\partial x^{2}}-\left(\frac{\partial g}{\partial x}\right)^{2}\right] \\
& -\sum_{i, j=1}^{N-1} h_{i j} \frac{\partial \mu_{i}}{\partial x} \frac{\partial \mu_{j}}{\partial x}-2 f_{N}^{2} \sigma_{N}^{2} \sum_{i, j=1}^{N-1} \frac{\partial^{2} h_{i j}}{\partial x^{2}} \\
& -2 \sum_{i=1}^{N-1} \frac{\partial^{2} h_{i i}}{\partial x^{2}} f_{i}^{2} \sigma_{i}^{2} .
\end{aligned}
$$

Similarly, E $\left\{\frac{\partial^{2}}{\partial y^{2}} \ln p(\tilde{\mathbf{z}} \mid \boldsymbol{l})\right\}$ can be obtained.

The off-diagonal terms in (32) can be obtained after some manipulation as

$$
\begin{aligned}
& \mathrm{E}\left\{\frac{\partial^{2}}{\partial x \partial y} \ln p(\tilde{\mathbf{z}} \mid \boldsymbol{l})\right\}=-\frac{(N-1)}{2 g^{2}}\left[g \frac{\partial^{2} g}{\partial x \partial y}-\frac{\partial g}{\partial x} \frac{\partial g}{\partial y}\right] \\
& \quad-\sum_{i, j=1}^{N-1} h_{i j} \frac{\partial \mu_{i}}{\partial x} \frac{\partial \mu_{j}}{\partial y}-2 f_{N}^{2} \sigma_{N}^{2} \sum_{i, j=1}^{N-1} \frac{\partial^{2} h_{i j}}{\partial x \partial y} \\
& \quad-2 \sum_{i=1}^{N-1} f_{i}^{2} \sigma_{i}^{2} \frac{\partial^{2} h_{i i}}{\partial x \partial y} .
\end{aligned}
$$

In addition, it can be shown from (18) and (2) that

$$
\begin{aligned}
& \frac{\partial \mu_{i}}{\partial x}=2\left(x_{i}-x_{N}\right), \\
& \frac{\partial \mu_{i}}{\partial y}=2\left(y_{i}-y_{N}\right) .
\end{aligned}
$$

Hence, $\sum_{i, j=1}^{N-1} h_{i j} \frac{\partial \mu_{i}}{\partial x} \frac{\partial \mu_{j}}{\partial x}$ becomes equal to $4 \mathbf{b}_{x}^{T} \boldsymbol{\Sigma}^{-1} \mathbf{b}_{x}$, where $\mathbf{b}_{x}\left(\mathbf{b}_{y}\right)$ is as given in Proposition 1. Similarly, $\sum_{i, j=1}^{N-1} h_{i j} \frac{\partial \mu_{i}}{\partial y} \frac{\partial \mu_{j}}{\partial y}$ and $\sum_{i, j=1}^{N-1} h_{i j} \frac{\partial \mu_{i}}{\partial x} \frac{\partial \mu_{j}}{\partial y}$ become equal to $4 \mathbf{b}_{y}^{T} \boldsymbol{\Sigma}^{-1} \mathbf{b}_{y}$ and $4 \mathbf{b}_{x}^{T} \boldsymbol{\Sigma}^{-1} \mathbf{b}_{y}$, respectively.

Then, from (34)-(36), the inverse of $\tilde{\mathbf{I}}$ in (32) can be calculated, and the CRLB given in Proposition 1 can be obtained.

\section{REFERENCES}

[1] J. J. Caffery, Wireless Location in CDMA Cellular Radio Systems. Boston: Kluwer Academic Publishers, 2000.

[2] S. Gezici, Z. Sahinoglu, H. Kobayashi, and H. V. Poor, "Ultra wideband geolocation," in Ultra Wideband Wireless Communications, H. Arslan and Z. N. Chen and M.-G. Di Benedetto (editors), Wiley-Interscience, 2006.

[3] S. Gezici, "A survey on wireless position estimation," Wireless Personal Communications, to appear, 2007.

[4] "IEEE 15-03-0489-03-004a-application-requirement-analysis-031127 v0.4." [Online]. Available: http://www.ieee802.org/15/pub/TG4.html

[5] S. Gezici, Z. Tian, G. B. Giannakis, H. Kobayashi, A. F. Molisch, H. V. Poor, and Z. Sahinoglu, "Localization via ultra-wideband radios: A look at positioning aspects for future sensor networks," IEEE Signal Processing Mag., vol. 22, no. 4, pp. 70-84, July 2005.

[6] IEEE P802.15.4a/D4 (Amendment of IEEE Std 802.15.4), "Part 15.4: Wireless medium access control (MAC) and physical layer (PHY) specifications for low-rate wireless personal area networks (LRWPANs)," July 2006.

[7] Z. Sahinoglu and S. Gezici, "Ranging for IEEE 802.15.4a ultrawideband devices," in Proc. IEEE Wireless and Microwave Technology Conference (WAMICON 2006), Clearwater, FL, Dec. 4-5 2006.

[8] K. Siwiak and J. Gabig, "IEEE 802.15.4IGa informal call for application response, contribution\#11," Doc.: IEEE 802.15-04/266r0, July, 2003. [Online]. Available: http://www.ieee802.org/15/pub/TG4a.html

[9] F. C. Commission, "Revision of the commissions rules to insure compatibility with enhanced 911 emergency calling systems," FCC Docket No. 94-102, July, 1996.

[10] J. J. Caffery and G. L. Stuber, "Overview of radiolocation in CDMA cellular systems," IEEE Commun. Mag., vol. 36, no. 4, pp. 38-45, Apr. 1998.

[11] F. Gustafsson and F. Gunnarsson, "Mobile positioning using wireless networks: Possibilites and fundamental limitations based on available wireless network measurements," IEEE Sig. Proc. Mag., vol. 22, no. 4, pp. 41-53, July 2005.

[12] W. Kim, J. G. Lee, and G. I. Jee, "The interior-point method for an optimal treatment of bias in trilateration location," IEEE Trans. Vehic. Technol., vol. 55, no. 4, pp. 1291-1301, July 2006.

[13] J. J. Caffery, "A new approach to the geometry of TOA location," in Proc. IEEE Vehic. Technol. Conf. (VTC), vol. 4, Boston, MA, Sep. 2000, pp. 1943-1949.

[14] S. Venkatesh and R. M. Buehrer, "A linear programming approach to NLOS error mitigation in sensor networks," in Proc. IEEE Int. Symp. Information Processing in Sensor Networks (IPSN), Nashville, Tennessee, Apr. 2006, pp. 301-308.

[15] Z. Li, W. Trappe, Y. Zhang, and B. Nath, "Robust statistical methods for securing wireless localization in sensor networks," in Proc. IEEE Int. Symp. Information Processing in Sensor Networks (IPSN), Los Angeles, CA, Apr. 2005, pp. 91-98.

[16] Y. T. Chan, H. Y. C. Hang, and P. C. Ching, "Exact and approximate maximum likelihood localization algorithms," IEEE Trans. Vehicular Technology, vol. 55, no. 1, pp. 10-16, Jan. 2006.

[17] I. Guvenc, C. C. Chong, and F. Watanabe, "Analysis of a linear leastsquares localization technique in LOS and NLOS environments," in Proc. IEEE Vehic. Technol. Conf. (VTC), Dublin, Ireland, Apr. 2007, pp. 1886-1890.

[18] Y. Qi, "Wireless geolocation in a non-line-of-sight environment," Ph.D. Dissertation, Princeton University, Dec. 2004.

[19] S. Al-Jazzar, J. J. Caffery, and H.-R. You, "A scattering model based approach to NLOS mitigation in TOA location systems," in Proc. IEEE Vehic. Technol. Conf. (VTC 2002), Birmingham, AL, May 2002, pp. 861-865.

[20] Y. Qi, H. Kobayashi, and H. Suda, "On time-of-arrival positioning in a multipath environment," IEEE Trans. Veh. Technology, vol. 55, no. 5, pp. 1516-1526, Sep. 2006.

[21] H. V. Poor, An Introduction to Signal Detection and Estimation. New York: Springer-Verlag, 1994.

[22] A. H. Sayed, A. Tarighat, and N. Khajehnouri, "Network-based wireless location," IEEE Signal Processing Mag., vol. 22, no. 4, pp. 24-40, July 2005. 